\title{
Prevalence of Malocclusion and Occlusal Traits in Children with Autism Spectrum Disorders
}

This article was published in the following Dove Press journal:

Clinical, Cosmetic and Investigational Dentistry

\section{Somaye Farmani \\ Shabnam Ajami \\ Neda Babanouri}

Department of Orthodontics, Orthodontic Research Center, School of Dentistry, Shiraz University of Medical Sciences, Shiraz, Iran
Correspondence: Neda Babanouri Department of Orthodontics, Orthodontic Research Center, School of Dentistry, Shiraz University of Medical Sciences, Qom Abad Blv, Ghasrodasht AV, Shiraz, Iran

Tel +98-7I3 6263193

Email nedababanouri508@protonmail. com
Background: The purpose of this cross-sectional study was to evaluate the prevalence of malocclusion in children with autism spectrum disorders (ASDs) and to determine the most common occlusal traits.

Materials and Methods: The present cross-sectional study was conducted among 7-15 years old children with ASDs and randomly selected healthy children with the same demographic characteristics. Molar relationship, lip incompetence, overbite, overjet, midline deviation, crossbite, and crowding/spacing were recorded. A descriptive analysis was performed for all variables. A comparison of the presence or absence of malocclusion traits between children with and without ASDs was assessed using the chi-square test. Univariate and multivariate logistic regressions were used to calculate odds ratio (OR) and 95\% confidence intervals $(95 \% \mathrm{CI})$ to assay various parameters between autism and non-autism group.

Results: A total of 96 patients were assessed, 47 patients in the ASD group, and 49 in the control (non-ASD) group. The results showed no significant difference between the ASD and control groups in terms of the overall prevalence of malocclusion $(P>0.05)$. However, the prevalence of increased overjet and Class II molar relationship was significantly higher in the ASD group compared to the control group $(\mathrm{P}=0.03)$. Patients in the control group showed a higher prevalence of midline deviation $(\mathrm{P}=0.001)$.

Conclusion: Despite a higher prevalence of overjet and Class II molar relationship in children with ASDs, the prevalence of malocclusion was not higher in this group of patients. Keywords: prevalence of malocclusion, autism spectrum disorders, disabled child, orthodontic care

\section{Introduction}

Autism spectrum disorders (ASDs) are a group of multifactorial neurodevelopmental conditions often accompanied by significant lifelong impairments. Typical behavioral symptoms of ASDs, in varying levels of severity, are impairments in social communication and interaction, and restricted and repetitive patterns of behavior. Its phenotype includes a range of symptoms in various areas, including cognitive, behavioral, affective, and sensory symptoms. ${ }^{1}$ ASDs affect individuals from different ethnic backgrounds and social classes, however, males are affected four to five times more often than females. ${ }^{2}$ In Iran, the prevalence of typical autism among 5 years old children has been reported at a rate of 6.2 per $10,000 .^{3}$ Although the exact etiology of ASDs is still unknown, genetic and environmental factors such as intrauterine viral infections, metabolic disorders, and exposure to teratogenic drugs are thought to be risk factors in the development of autism. ${ }^{4}$ 
Several studies have been conducted on periodontal status and dental caries formation in patients with ASD. Some studies have reported that such patients tend to have unhealthier periodontal conditions and poorer oral hygiene. ${ }^{5-8}$ Whereas some others reported no significant difference in the prevalence of dental caries between individuals with and without autism. ${ }^{9,10}$ Some other studies reported that the frequency and severity of dental caries were low in a group of patients with ASD. ${ }^{7,11}$

The prevalence of malocclusion and oral habits in children with ASDs has been evaluated in some studies, though the results were contradictory. In comparison with non-ASD patients, it has been shown that the prevalence of oral habits (bruxism, tongue thrust, and thumb sucking) was significantly higher in patients with ASD. ${ }^{12,13}$ Ozgen et al assessed morphological features in children with ASDs and reported a greater tendency toward certain morphological traits such as anterior open bite. ${ }^{14}$ However, another study found no statistically significant differences between the ASD and non-ASD groups in terms of open bite; interestingly, dental crowding was lower in the ASD group. ${ }^{15}$ The results of another study revealed an insignificant increase in the prevalence of deep overbite, increased overjet, and anterior crossbite in patients with ASD. ${ }^{1}$ A recent study among Canadian children with ASDs showed a significant increase in the prevalence of severe maxillary crowding, posterior crossbite, and increased overjet in their ASD group. ${ }^{2}$ Aljubour et al determined the characteristics of primary dentition occlusion among preschool children with ASD and showed that most children in the study group exhibited a distal step molar relationship, a Class II canine relationship, and increased overjet and overbite. ${ }^{16}$

Our literature review also confirmed discrepancies in the reported malocclusion patterns in children with ASDs. Furthermore, we found no studies on the prevalence of malocclusion in Iranian children with autism. Besides, due to the lack of communication and bonding abilities in children with autism, the potential for poor cooperation during the orthodontic treatment, orthodontic management of this group of patients could be a challenge. It is known that early diagnosis of developing malocclusion allows opting for more preventive strategies and non-complex interceptive treatments. Hence, the main objective of the present study was to determine the prevalence of malocclusion and to establish the most common malocclusion patterns in Iranian children with ASDs.

\section{Materials and Methods}

The present cross-sectional study was approved by the Ethical Committee of Shiraz University of Medical Sciences, Shiraz, Iran (Code:IR.SUMS.REC.1397.398) and conducted from June 2018 to October 2018. Written informed consent was obtained from the parents of the children who participated in the study.

The target population was children with a medical diagnosis of ASDs referred to the three main rehabilitation centers in Shiraz, Iran (seventeen children from FrooghAsr Rehabilitation Center, fourteen children from Shiraz School of Autism and sixteen children from Autism Care Center). The study groups included children with ASDs (ASD group) and healthy children (control group) with the same demographic characteristics. The inclusion criteria for participation in the ASD group were: age 7-15 years, confirmed medical diagnosis of ASDs, and children in the mixed or permanent dentition stage. The inclusion criteria for participation in the control group were aged 7-15 years old and healthy children in the mixed or permanent dentition stage. The exclusion criteria for both groups were an incomplete eruption of upper and lower incisors, history of previous orthodontic treatment, presence of any craniofacial anomalies (cleft lip and palate), or syndromes other than ASDs.

An orthodontic resident who was trained and calibrated by an expert orthodontist with more than 10 years of experience, was assigned to examine the children and collect the required data. A mouth mirror, ruler, and a sliding digital caliper (Digimatic ${ }^{\circledR}$, Mitutoyo UK Ltd, Andover, Hampshire, UK) were used in the examinations. For every child, a registration chart related to malocclusion was designed, which included parameters such as transverse, sagittal, and vertical dimensions and dental alignment. The subjects were in an upright position while their head was in a natural position during the examination time. $^{17}$

To quantify measurement errors, 20 children (10 ASD group, 10 Control group), were re-examined within two weeks. To detect intraexaminer reliability, the intraclass correlation coefficient (ICC) reliability index was used.

\section{Transverse Dimension}

Crossbite was registered when buccal cusps of at least one of the maxillary posterior teeth (premolars and molars) occluded lingually or edge-to-edge to the buccal cusps of the mandibular teeth. Scissor bite was registered when the 
lingual surface of any of the maxillary posterior teeth occluded with the buccal surface of the mandibular antagonist teeth. The discrepancy between the upper and lower dental midlines was assessed and measured in millimeters (mm). No judgment was made regarding the position of dental midline relative to the facial midline. A midline discrepancy of more than $2 \mathrm{~mm}$ was recorded as midline deviation.

\section{Sagittal Dimension}

The Angel's classification was used to register the molar relationship (Class I, Class II, and Class III). A half unit Class II or Class III has registered as Class II or Class III malocclusion, respectively. Overjet was recorded as the horizontal distance $(\mathrm{mm})$ between the most labial point of the incisor edge of the upper incisor and the most labial surface of the corresponding lower incisors. A distance less than $1 \mathrm{~mm}$ or greater than $4 \mathrm{~mm}$ was considered as a decrease or increase in overjet, respectively. Anterior crossbite was also registered as a decreased overjet.

\section{Vertical Dimension}

Overbite was considered as a vertical overlap of the maxillary central incisors over the mandibular central incisors when the posterior teeth were in the maximum intercuspation. The overbite was classified as normal (5-25\% coverage), increased (more than 25\% coverage), and decreased (less than $5 \%$ coverage). The open bite was considered as a decreased overbite. Lip incompetence was registered at an interlabial gap of more than $4 \mathrm{~mm}$.

\section{Dental Alignment}

The degree of spacing and crowding was calculated by comparing the available space (from the mesial surface of the first molar to the other) and the space required for proper alignment with the corresponding teeth. For patients in the mixed dentition stage, the size of unerupted permanent teeth was estimated using the Moyers prediction tables at a $65 \%$ probability. Dental space was categorized as normal (no spacing or crowding more than $1 \mathrm{~mm}$ ) and abnormal (space deficiency or spacing more than $1 \mathrm{~mm}$ ). Any tooth movement due to previous extractions were taken into account.

The criteria for defining a normal occlusion were a Class I molar relationship, a positive overjet (1-4 mm), a positive overbite ( $5-25 \%$ coverage), lip competence, no posterior crossbite, or midline discrepancy, and wellaligned teeth.

\section{Statistical Analysis}

Data analysis was performed using SPSS software version 18.0 (Chicago, IL, USA). A descriptive analysis was performed for all variables. A comparison of the presence or absence of malocclusion traits between children with and without ASDs was assessed using the chi-square test. Univariate and multivariate logistic regressions were used to calculate odds ratio (OR) and $95 \%$ confidence intervals $(95 \% \mathrm{CI})$ to assay various parameters between autism and non-autism group. Statistical significance was based on probability values of 0.05 or less.

\section{Results \\ Study Error}

The result of the ICC reliability index showed significant consistency between the main and repeated records $(\mathrm{P}<0.001)$.

A total of 96 children were assessed, divided into a control group $(n=49)$ and ASD group $(n=47)$. The age range of the participants varied from 8.13 to 13.72 years and the majority were boys (Table 1). There was no statistically significant difference between the study groups in terms of age and sex $(\mathrm{P}>0.05)$. The overall prevalence of malocclusion was $79.2 \%$ and $76.1 \%$ in the control and ASD group, respectively. The results showed no significant difference in the overall prevalence of malocclusion between the study groups $(\mathrm{P}=0.45)$.

The most prevalent malocclusions were increased overjet and Class II molar relationship in the ASD group and midline discrepancy in the control group. The results showed a significantly higher prevalence of midline deviation in the control group compared to the ASD group $(\mathrm{P} \leq 0.001)$. There was a significantly higher prevalence of

Table I Demographic Characteristics and Prevalence of Malocclusion of Children with and without ASD

\begin{tabular}{|c|l|l|}
\hline Variable & $\begin{array}{l}\text { ASD } \\
\text { N=47 }\end{array}$ & $\begin{array}{l}\text { Non-ASD } \\
\mathbf{N}=49\end{array}$ \\
\hline $\begin{array}{c}\text { Sex } \\
\text { Male }\end{array}$ & $36(76.6 \%)$ & $28(57.1 \%)$ \\
Female & II (23.4\%) & $21(42.9 \%)$ \\
Age (y) & $10.74 \pm 2.1$ & $9.5 \pm 1.32$ \\
\hline $\begin{array}{l}\text { Malocclusion } \\
\text { No }\end{array}$ & & \\
Yes & II (23.9\%) & $10(20.8 \%)$ \\
\hline
\end{tabular}


increased overjet and Class II malocclusion in the ASD group compared to the control group $(\mathrm{P}=0.03)$. Children in the ASD group were 6 times more likely to have increased overjet than those in the control group (OR: 6, 95\% CI: 1.74-20.59). There was no statistically significant difference between the two groups in terms of crossbite, lip competency, overbite, and space analysis. However, the difference between the groups in terms of lip incompetency tended to be significant (ASD: $19.1 \%$, control: $6.3 \%$, $\mathrm{P}=0.07$ ). (Tables 2,3 and 4)

\section{Discussion}

Despite a high prevalence of ASDs in children, only a few studies have compared the dentoskeletal characteristics of this population with those of healthy individuals; moreover, without unanimity on the prevalence of malocclusion. The present cross-sectional study was conducted among Iranian children with ASDs (7-15 years old) to provide preliminary information on the prevalence of malocclusion. This age group was specially selected since optimal interceptive orthodontic treatment results can be

Table 2 Distribution of Malocclusions in Children with and without ASD Based on Sagittal Dimension

\begin{tabular}{|l|l|l|l|l|}
\hline Variable & ASD & Non-ASD & P value & Odds Ratio \\
\hline Angle classification & & & & $2.05(0.905-4.654)$ \\
Class I & $20(55.6 \%)$ & $30(88.2 \%)$ & & \\
Class II & $16(44.4 \%)$ & $4(11.8 \%)$ & 0.03 & \\
Class III & $7(25.9 \%)$ & $13(30.2 \%)$ & 0.79 & $2.05(0.905-4.654)$ \\
\hline Overjet & & & & \\
Normal & $20(55.6 \%)$ & $30(88.2 \%)$ & 0.03 & \\
Increased & $16(44.4 \%)$ & $4(11.8 \%)$ & 0.79 & \\
Decreased & $7(25.9 \%)$ & $13(30.2 \%)$ & & \\
\hline
\end{tabular}

Table 3 Distribution of Malocclusions in Children with and without ASD Based on Vertical Dimension

\begin{tabular}{|l|l|l|l|l|}
\hline Variable & ASD & Non-ASD & P value & Odds Ratio \\
\hline Overbite & & & & $0.920(0.413-2.049)$ \\
Normal & $24(66.7 \%)$ & $24(75 \%)$ & 0.59 & \\
Increased & $12(33.3 \%)$ & $8(25 \%)$ & 0.29 & $3.355(0.897-14.067)$ \\
Decreased & $7(22.6 \%)$ & $13(35.1 \%)$ & & \\
\hline Lip & & & & \\
Competent & $38(80.9 \%)$ & $45(93.8 \%)$ & 0.07 & \\
Incompetent & $9(19.1 \%)$ & $3(6.3 \%)$ & & \\
\hline
\end{tabular}

Table 4 Distribution of Malocclusions in Children with and without ASD Based on Transverse Dimension and Space Analysis

\begin{tabular}{|l|l|l|l|l|}
\hline Variable & ASD & Non-ASD & P value & Odds Ratio \\
\hline $\begin{array}{l}\text { Midline deviation } \\
\text { Coincident } \\
\text { Deviated }\end{array}$ & $\begin{array}{l}38(82.6 \%) \\
8(17.4 \%)\end{array}$ & $\begin{array}{l}21(43.8 \%) \\
27(56.3 \%)\end{array}$ & $0.178(0.07 \mathrm{I}-0.446)$ \\
\hline $\begin{array}{l}\text { Crossbite } \\
\text { No }\end{array}$ & $\begin{array}{l}4(93.6 \%) \\
\text { Anterior+ Posterior }\end{array}$ & $\begin{array}{l}42(85.7 \%) \\
7(14.3 \%)\end{array}$ & $0.00 \mathrm{I}$ & $0.3 \mathrm{I}$ \\
\hline $\begin{array}{l}\text { Space analysis } \\
\text { Normal } \\
\text { Abnormal (crowding +spacing) }\end{array}$ & $39(86.7 \%)$ & $6(13.3 \%)$ & $38(77.6 \%)$ & $0.099-1.687)$ \\
\hline
\end{tabular}


achieved at a younger age. Our results showed no significant difference in the prevalence of malocclusion between ASD and control groups. This finding was in line with previous studies reporting similar malocclusion and orthodontic treatment needs in children with and without ASD. ${ }^{5,18}$ In contrast, another study reported a higher prevalence of malocclusion in Canadian children with ASD compared to healthy children. ${ }^{2}$

A higher prevalence of malocclusion in the ASD group was associated with increased overjet and Class II molar relationship. Increased overjet among children with ASDs was also reported in previous studies. ${ }^{2,18}$ This could be due to the para-functional use of the mouth, tongue, and jaw by patients with ASD. Oral habit behavior in such patients includes pacifier sucking, bruxism, tongue thrust, lip sucking and biting, pica, and self-injurious habits. ${ }^{19,20}$ A previous study reported that children with such oral habits are susceptible to malocclusion traits like excessive overjet, anterior open bite, and posterior crossbite. ${ }^{21}$ In the present study, the para-functional oral habit in children with ASD was not addressed. Nonetheless, we hypothesized that the similarities between malocclusion traits and oral habits in such patients are indicative of their correlation. Further research is required to substantiate this hypothesis.

Our results showed that the prevalence of lip incompetency tended to be significantly higher in the ASD group compared to the control group. Although previous studies did not measure lip incompetency, Ozgen et al found that short philtrum and open-mouth expression were significantly higher in patients with ASD compared to normal individuals. ${ }^{14}$

In line with a study by Kuter et al, ${ }^{15}$ we found no difference between our study groups in terms of open bite and crossbite. In contrast, a previous study reported a significant increase in anterior open bite and a tendency to posterior crossbite malocclusion in patients with ASD. ${ }^{2}$ These inconsistencies could be due to the lack of standardized nomenclature, the absence of uniform diagnostic criteria, and/or differences in age and ethnic background of the target population.

According to the American Centers for Disease Control and Prevention, about 1 in 100 children aged 8 years old suffer from ASD. ${ }^{22}$ This means that orthodontists will inevitably face the challenges associated with treating these children. Children with ASD suffer from reduced comprehension, higher levels of anxiety, short attention span, low frustration tolerance, and reduced coordination of upper limb movement. Consequently, they are unable to remain seated in a dental chair and thus become uncooperative. All these combined with an exaggerated gag reflex pose a challenge to orthodontists during routine orthodontic treatment. ${ }^{23}$ It is therefore essential that orthodontists have a good understanding of these challenges to plan an effective treatment of malocclusion in these children. On the other hand, interaction with the parents is important to identify and implement the best approach to manage these children during the treatment. ${ }^{24}$ Hence orthodontists must gain the confidence of the parents and encourage their support. Various techniques could be used to communicate with and manage uncooperative children. For instance, the presence of the parents during treatment, oral commands (in the form of short, clear, and simple sentences), the Tell-Show-Do method, behavior modification techniques, and positive reinforcement could be used. Orthodontists should consider the use of these techniques as part of the treatment process through gradual introduction during several visits before starting the actual treatment. ${ }^{25-28}$ The desensitization technique could also be used to manage autistic children. This technique consists of the gradual introduction of orthodontic tools and treatment environment during multiple visits to gradually relieve the stress and anxiety in these children. ${ }^{24}$ Orthodontic procedures should also be evaluated beforehand, particularly the use of protective stabilization devices to restrain the patients and to manage their behavior. As a last resort, sedation or general anesthesia could be considered.

Orthodontists should leverage on the repetitive behavioral traits of children with ASD through repeated presentation and fitting removable appliances (ie, visual pedagogy). This allows autistic children to gain confidence in using these appliances, which in turn results in a better orthodontic outcome. In line with previous studies, we recommend the use of removable orthodontic appliances in autistic children. These appliances should be as small as possible and reinforced by wires since hyperesthesia is common in autistic children. ${ }^{29,30}$

\section{Study Limitations}

The results of this study should be considered with caution due to the small number of participants and the cross-sectional nature of the study, which makes it difficult to establish an association between the risk factors and malocclusion traits. Besides, data about oral habits did not collect, so the cause and effect relationship could 
not be established. Moreover, the investigation was conducted in only one city in Iran, so the finding cannot be generalized. However, Shiraz is the largest city in the south area of Iran and is likely to have similar characteristics to the other cities. Further studies with a larger sample size and evaluation of possible factors that may be responsible for the increased prevalence of malocclusion traits in autistic children are encouraged. Moreover, information about oral habits was lacking; Additionally, the study was conducted in only 1 pediatric hospital in Montreal, and its findings cannot be generalized. Future research should be encouraged to explore the factors that may be involved in the increased prevalence of malocclusion traits among children with ASD.

\section{Conclusion}

There was no significant difference between patients with and without ASD in terms of the overall prevalence of malocclusion. However, the prevalence of increased overjet and Class II molar relationship was higher in patients with ASDs.

\section{Acknowledgment}

The authors thank the Vice-chancellor of the Shiraz University of Medical Science for supporting this research (Grant\#16499). The authors also thank Dr. Vossoughi of the Center for Research Improvement of the School of Dentistry for the statistical analysis.

\section{Funding}

The study was financially supported by the Vice Chancellery of Shiraz University of Medical Sciences, Shiraz, Iran (grant number: 16499).

\section{Disclosure}

The authors report no conflicts of interest in this work.

\section{References}

1. Wiggins LD, Levy SE, Daniels J, et al. Autism spectrum disorder symptoms among children enrolled in the Study to Explore Early Development (SEED). $J$ Autism Dev Disord. 2015;45 (10):3183-3194. doi:10.1007/s10803-015-2476-8

2. Fontaine-Sylvestre C, Roy A, Rizkallah J, Dabbagh B, Ferraz Dos Santos B. Prevalence of malocclusion in Canadian children with an autism spectrum disorder. Am J Orthod Dentofacial Orthop. 2017;152 (1):38-41. doi:10.1016/j.ajodo.2017.02.014

3. Samadi SA, Mahmoodizadeh A, McConkey R. A national study of the prevalence of autism among five-year-old children in Iran. Autism. 2012;16(1):5-14. doi:10.1177/1362361311407091
4. Johnson CP, Myers SM. Identification and evaluation of children with autism spectrum disorders. Pediatrics. 2007;120(5):1183-1215. doi:10.1542/peds.2007-2361

5. Luppanapornlarp S, Leelataweewud P, Putongkam P, Ketanont S. Periodontal status and orthodontic treatment need of autistic children. World J Orthod. 2010;11(3):256-261.

6. Jaber MA. Dental caries experience, oral health status and treatment needs of dental patients with autism. J Appl Oral Sci. 2011;19 (3):212-217. doi:10.1590/S1678-77572011000300006

7. Orellana LM, Silvestre FJ, Martinez-Sanchis S, Martinez-Mihi V, Bautista D. Oral manifestations in a group of adults with autism spectrum disorder. Med Oral Patol Oral Cir Bucal. 2012;17(3): e415-9. doi:10.4317/medoral.17573

8. Vishnu Rekha C, Arangannal P, Shahed H. Oral health status of children with autistic disorder in Chennai. Eur Arch Paediatr Dent. 2012;13(3):126-131. doi:10.1007/BF03262858

9. Bassoukou IH, Nicolau J, Dos Santos MT. Saliva flow rate, buffer capacity, and pH of autistic individuals. Clin Oral Investig. 2009;13 (1):23-27. doi:10.1007/s00784-008-0209-5

10. Rai K, Hegde AM, Jose N. Salivary antioxidants and oral health in children with autism. Arch Oral Biol. 2012;57(8):1116-1120. doi:10.1016/j.archoralbio.2012.03.006

11. Loo CY, Graham RM, Hughes CV. The caries experience and behavior of dental patients with autism spectrum disorder. J Am Dent Assoc. 2008;139(11):1518-1524. doi:10.14219/jada.archive.2008.0078

12. Medina AC, Sogbe R, Gomez-Rey AM, Mata M. Factitial oral lesions in an autistic paediatric patient. Int $J$ Paediatr Dent. 2003;13(2):130-137. doi:10.1046/j.1365-263X.2003.00440.x

13. Buyukbayraktar ZC, Doruk C. Orthodontic approach to patients with autism: a review. Turk J Orthod. 2019;32(3):172-175. doi:10.5152/ TurkJOrthod.2019.18078

14. Ozgen H, Hellemann GS, Stellato RK, et al. Morphological features in children with autism spectrum disorders: a matched case-control study. J Autism Dev Disord. 2011;41(1):23-31. doi:10.1007/s10803010-1018-7

15. Kuter B, Guler N. Caries experience, oral disorders, oral hygiene practices and socio-demographic characteristics of autistic children. Eur J Paediatr Dent. 2019;20(3):237-241. doi:10.23804/ejpd.2019. 20.03.13

16. Aljubour A, Al-Sehaibany F. Characteristics of primary dentition occlusion among preschool children with autism spectrum disorder. $J$ Dent Health Oral Disord Ther. 2018;9(4):262-266.

17. Okeson J, Graber L, Vanarsdall R, Vig K, Huang G Orthodontics: current principles and techniques. 2017.

18. Du RY, Yiu CK, King NM, Wong VC, McGrath CP. Oral health among preschool children with autism spectrum disorders: a case-control study. Autism. 2015;19(6):746-751. doi:10.1177/ 1362361314553439

19. Sarnat H, Samuel E, Ashkenazi-Alfasi N, Peretz B. Oral health characteristics of preschool children with autistic syndrome disorder. J Clin Pediatr Dent. 2016;40(1):21-25. doi:10.17796/ 1053-4628-40.1.21

20. El Khatib AA, El Tekeya MM, El Tantawi MA, Omar T. Oral health status and behaviours of children with Autism Spectrum Disorder: a case-control study. Int $J$ Paediatr Dent. 2014;24(4):314-323. doi:10.1111/ipd.12067

21. Warren JJ, Bishara SE, Steinbock KL, Yonezu T, Nowak AJ. Effects of oral habits' duration on dental characteristics in the primary dentition. J Am Dent Assoc. 2001;132(12):1685-93; quiz 726. doi:10.14219/jada.archive.2001.0121

22. Hernandez P, Ikkanda Z. Applied behavior analysis: behavior management of children with autism spectrum disorders in dental environments. J Am Dent Assoc. 2011;142(3):281-287. doi:10. 14219/jada.archive.2011.0167

23. Becker A, Shapira J, Chaushu S. Orthodontic treatment for the special needs child. Prog Orthod. 2009;10(1):34-47. 
24. Nelson TM, Sheller B, Friedman CS, Bernier R. Educational and therapeutic behavioral approaches to providing dental care for patients with Autism Spectrum Disorder. Spec Care Dentist. 2015;35(3):105-113. doi:10.1111/scd.12101

25. Ozsoy OP, Bingol SI. Extraction orthodontic treatment in an autistic patient. Turk J Orthod. 2017;30(1):28-32. doi:10.5152/TurkJOrthod. 2017.0013

26. Murshid EZ. Effectiveness of a preparatory aid in facilitating oral assessment in a group of Saudi children with autism spectrum disorders in Central Saudi Arabia. Saudi Med J. 2017;38(5):533-540. doi:10.15537/smj.2017.5.17398

27. Salyer KE, Rozen SM, Genecov ER, Genecov DG, editors. Unilateral cleft lip-approach and technique. Seminars in plastic surgery; 2005: Copyright $(2005$ by Thieme Medical Publishers, Inc.; Seventh Avenue, New .... 333.
28. Delli K, Reichart PA, Bornstein MM, Livas C. Management of children with autism spectrum disorder in the dental setting: concerns, behavioural approaches and recommendations. Med Oral Patol Oral Cir Bucal. 2013;18(6):e862-868. doi:10.4317/medoral.19084

29. Saito K, Jang I, Kubota K, et al. Removable orthodontic appliance with nickel-titanium spring to reposition the upper incisors in an autistic patient. Spec Care Dentist. 2013;33(1):35-39. doi:10.1111/ j.1754-4505.2012.00291.x

30. Alqahtani H. Medically compromised patients in orthodontic practice: review of evidence and recommendations. Int Orthod. 2019;17 (4):776-788. doi:10.1016/j.ortho.2019.08.015

\section{Publish your work in this journal}

Clinical, Cosmetic and Investigational Dentistry is an international, peer-reviewed, open access, online journal focusing on the latest clinical and experimental research in dentistry with specific emphasis on cosmetic interventions. Innovative developments in dental materials, techniques and devices that improve outcomes and patient satisfaction and preference will be highlighted. The manuscript management system is completely online and includes a very quick and fair peer-review system, which is all easy to use. Visit http://www.dovepress.com/testimonials.php to read real quotes from published authors. 